\title{
openheart Copeptin in patients with heart failure and preserved ejection fraction: a report from the prospective KaRen-study
}

To cite: Hage C, Lund LH, Donal $\mathrm{E}$, et al. Copeptin in patients with heart failure and preserved ejection fraction: a report from the prospective KaRen-study. Open Heart 2015;2:e000260.

doi:10.1136/openhrt-2015000260

Received 3 March 2015 Revised 8 September 2015 Accepted 13 October 2015

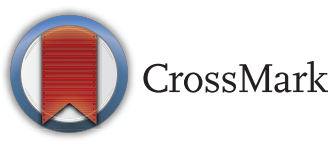

${ }^{1}$ Department of Cardiology, Karolinska University Hospital, Stockholm, Sweden ${ }^{2}$ Département de Cardiologie, Centre Hospitalier Universitaire de Rennes, Rennes, France

Correspondence to Camilla Hage; camilla.hage@karolinska.se

\section{ABSTRACT}

Introduction: Underlying mechanisms of heart failure (HF) with preserved ejection fraction (HFPEF) remain unknown. We explored copeptin, a biomarker of the arginine vasopressin system, hypothesising that copeptin in HFPEF is elevated, associated with diastolic dysfunction and N-terminal pro-brain natriuretic peptide (NT-proBNP) and predictive of HF hospitalisation and mortality.

Methods and analysis: In a prospective observational substudy of the The Karolinska Rennes (KaRen) 86 patients with symptoms of acute HF and ejection fraction $(E F) \geq 45 \%$ were enrolled. After 4-8 weeks, blood sampling and echocardiography was performed. Plasma-copeptin was analysed in 86 patients and 62 healthy controls. Patients were followed in median 579 days (quartile 1; quartile 3 (Q1;Q3) 276;1178) regarding the composite end point all-cause mortality or HF hospitalisation.

Ethics and dissemination: The patients with HFPEF had higher copeptin levels, median $13.56 \mathrm{pmol} / \mathrm{L}$ (Q1; Q3 8.56;20.55) than controls $5.98 \mathrm{pmol} / \mathrm{L}(4.15 ; 9.42$; $\mathrm{p}<0.001$ ). Diastolic dysfunction, assessable in $75 / 86$ patients, was present in 45 and absent in 30 patients. Copeptin did not differ regarding diastolic dysfunction and did not correlate with cardiac function but with NT-proBNP ( $r=0.223$; $p$ value $=0.040)$. In univariate Cox regression analysis log copeptin predicted the composite end point (HR 1.56 (95\% Cl 1.03 to 2.38; $\mathrm{p}$ value $=0.037)$ ) but not after adjusting for NT-proBNP (HR 1.39 (95\% Cl 0.91 to 2.12; p value $=0.125)$ ).

Conclusions: In the present patients with HFPEF, copeptin is elevated, correlates with NT-proBNP but not markers of diastolic dysfunction, and has prognostic implications, however blunted after adjustment for NT-proBNP. The HFPEF pathophysiology may be better reflected by markers of neurohormonal activation than by diastolic dysfunction.

Trial registration number: ClinicalTrials.gov NCT00774709.

\section{INTRODUCTION}

Patients with heart failure (HF) and a preserved ejection fraction (HFPEF) constitute nearly $50 \%$ of the $\mathrm{HF}$ population and HFPEF is associated with a decreased life

\section{KEY QUESTIONS}

What is already known about this subject?

- Copeptin has been shown to be prognostic predictor of heart failure (HF) hospitalisation and mortality in acute HF with no knowledge of whether patients have reduced (HFREF) or preserved ejection fraction (HFPEF)

What does this study add?

- We demonstrate that in patients with HFPEF copeptin is elevated, correlates with N-terminal pro-brain natriuretic peptide but not with markers of diastolic dysfunction and is a prognostic predictor.

How might this impact on clinical practice?

- Knowledge on the underlying mechanisms in HFPEF is crucial for the much needed development of novel treatment options for these patients.

expectancy. ${ }^{1}$ Although often elderly and predominately of female gender, this is a heterogeneous group of patients with diverse comorbidities such as hypertension, atrial fibrillation, diabetes and obesity, and the definition and diagnostic tools remain controversial. $^{2-6}$ The impaired prognosis is, however, not fully explained by these conditions as higher mortality rates have been reported in patients with HFPEF than in patients with similar age and gender profile and with similar comorbidities but without the HFPEF syndrome. ${ }^{7}$

In HF several hormonal systems are suggested to be involved. One of these is the arginine vasopressin system, primarily activated by increased osmolality followed by hypovolemia, leading to vasoconstriction and water retention. In patients with $\mathrm{HF}$ and reduced ejection fraction (HFREF), levels of vasopressin are elevated compared to controls and correlated with disease severity. Vasopressin is difficult to measure due to its in vitro instability and rapid clearance from 
the circulation. Therefore, copeptin, the stable C-terminal part of the prohormone, synthesised and released in equimolar amounts with vasopressin, is easier to measure in blood and accurately reflects vasopressin activation. $^{9}$

In acute HF with no knowledge of whether left ventricular function is depressed or not copeptin has been shown to be prognostic predictor of HF hospitalisation and mortality. ${ }^{10-12}$ In contrast, the role of the vasopressin system in patients with HFPEF is still largely unexplored. Therefore, increased understanding of its role may potentially elucidate the pathophysiological process and thus contribute to the development of new treatment strategies in HFPEF, where guideline indicated interventions are lacking. ${ }^{13}$

The present study aimed to test the hypothesis that copeptin in HFPEF is (1) elevated, (2) associated with diastolic dysfunction and N-terminal pro-brain natriuretic peptide (NT-proBNP) and (3) predictive of HF hospitalisation and mortality.

\section{MATERIAL AND METHODS}

This is a substudy of the The Karolinska Rennes (KaRen) which was a prospective observational multicenter study characterising patients with HFPEF. A detailed description of the protocol has been presented elsewhere. ${ }^{14}$ In brief, 539 patients presenting to the hospital with acute signs and symptoms of $\mathrm{HF}$ according to the Framingham criteria, NT-proBNP $>300 \mathrm{ng} / \mathrm{L}$ and a left ventricular ejection fraction (LVEF) $\geq 45 \%$ were enrolled in French and Swedish centres. The patients returned to the hospital in stable condition 4-8 weeks after enrolment for a follow-up visit including blood sampling and echocardiography. Changes in clinical characteristics have previously been presented. ${ }^{15}$ The KaRen biochemistry substudy was prespecified and included Swedish centres only. A number of 86 patients were recruited 21 May 2007 to 29 December 2011 at Karolinska University Hospital and were according to the protocol thereafter followed until 30 September 2012 when vital status was assessed by telephone contact or by the Swedish National Patient Register and Population Register. The primary outcome was according to the main study protocol defined as time to mortality from any cause or first hospitalisation due to HF. All HF hospitalisations were adjudicated and defined according to clinical judgment by the local investigator. The secondary outcome was time to mortality from any cause.

At the follow-up visit blood samples were collected in a fasting condition in the morning in ethylenediaminetetraacetic acid tubes, centrifuged and plasma was stored in aliquots in $-70^{\circ} \mathrm{C}$ until analysis. Additionally copeptin was analysed, in the same laboratory with same method in a population-based healthy control material $(n=62)$ matched for age within 1 year. ${ }^{16}$ Copeptin was analysed with a commercial available automated immunofluorescent assay (us Kryptor Compact Plus, BRAMHS,
Henningsdorf/Berlin, Germany). ${ }^{17} 18$ The lower detection limit of $0.9 \mathrm{pmol} / \mathrm{L}$ and interassay coefficients of variation $18.3 \%$ for $1.4 \mathrm{pmol} / \mathrm{L}, 6.8 \%$ for $9.3 \mathrm{pmol} / \mathrm{L}$ and less than $3 \%$ for concentrations $>18 \mathrm{pmol} / \mathrm{L}{ }^{18}$

The range of copeptin in healthy individuals has been published previously and is expressed as median (2.5th97.5th centiles); 4.2 (1.7-11.25) $\mathrm{pmol} / \mathrm{L}{ }^{9}{ }^{9}$ NT-proBNP was analysed by Elecsys electrochemiluminescence 'sandwich' immunoassay, proBNPII (Roche Diagnostics, Bromma, Sweden) with a lower detection limit of $5 \mathrm{ng} /$ $\mathrm{L}$ and interassay coefficients of variation of $\leq 20 \%$. ${ }^{19}$ Creatinine was analysed by an enzymatic reaction with a modified rate Jaffe-method (Beckman Coulter, SYNCHRON system) and glomerular filtration rate (GFR) was calculated according to the Chronic Kidney Disease Epidemiology Collaboration (CKD-EPI) formula. $^{20}$

The echocardiographic assessment was performed on a VIVID 7 echo-platform (GE VingMed, Horten, Norway) and stored in a raw-data format for off-line central analysis on Echopac PC BT12 instrumentation and software (GE Healthcare) at the Rennes University Centre for Clinical Research, France. All measurements were performed according to the recommendations of the American and European Societies of echocardiography with respect to the cardiac chamber and right heart measurements. Each examination was interpreted once and measurements were performed three times and averaged by an echocardiographist (ED) blinded to the specific clinical history of the patient.

To fulfil the definition of diastolic dysfunction two of three parameters related to diastolic function in the ESC guidelines were required. Diastolic dysfunction was defined as either left atrial volume index (LAVI; calculated as left atrial volume in $\mathrm{mL}$ divided by body surface area in $\mathrm{m}^{2}$ ) $>34 \mathrm{~mL} / \mathrm{m}^{2}$ or left ventricular hypertrophy (LVH) assessed as left ventricular mass index (LVMI; calculated as left ventricular mass divided by body surface area) $>95 \mathrm{~g} / \mathrm{m}^{2}$ in females and $>115 \mathrm{~g} / \mathrm{m}^{2}$ in males in addition to either ratio of early transmitral velocity to mitral annular early velocity $\left(\mathrm{E} / \mathrm{e}^{\prime}\right)>15$ or $\mathrm{E}^{\prime}<9 \mathrm{~cm} / \mathrm{s}$, according to the European Society of Cardiology guidelines. ${ }^{21}$ Additionally cardiac function was assessed as LVEF, left ventricular end diastolic diameter (LVEDd), isovolumetric relaxation time (IVRT) and E-wave deceleration time.

Continuous variables were expressed as median and quartile 1 and quartile $3(\mathrm{Q} 1 ; \mathrm{Q} 3)$ and the Wilcoxon rank sum test was used to determine differences between groups. Categorical variables were expressed as numbers and percentages and analysed using Fisher's exact test. Correlations between copeptin and echocardiographic measurements of cardiac function were determined using Spearman's correlation coefficient. In addition correlations were assessed between copeptin and selected clinical and biochemical characteristics (age, body mass index (BMI), NT-proBNP and GFR) selected as they may influence copeptin levels and/or outcome in HFPEF. 
The role of copeptin as a predictor of the primary and secondary outcomes was analysed by Cox proportional hazards model and presented as HR and 95\% CI. The same variables as in the correlation analyses were used as covariates in multivariable Cox regression models (presented in table 1). In the final multivariable model three clinically significant covariates, age, gender and NT-proBNP were included. Copeptin and NT-proBNP were log-transformed prior to analysis. All $\mathrm{p}$ values were two-sided and statistical significance was set at 0.05 . All statistical analyses were performed using SAS software V.9.3 (SAS Institute, Cary, North Carolina, USA).

The KaRen study was conducted according to International Conference on Harmonisation and Good Clinical Practice guidelines and the investigation conforms with the principles outlined in the Declaration of Helsinki. The biochemistry substudy was approved by the ethical review board at Karolinska Institutet. Written and oral informed consent was obtained from all patients prior to enrolment, including a separate consent regarding the subtudy.

\section{RESULTS}

Characteristics of all 86 patients with HFPEF divided according to presence of diastolic dysfunction are presented in table 2. In median patients were 73 years old and $51 \%$ were females. In all patients cardiac function assessed as ejection fraction was $64 \%(58 ; 68), \mathrm{E} / \mathrm{e}^{\prime}$ ratio was $10.8(8.3 ; 14.0)$ and LAVI $43 \mathrm{~mL} / \mathrm{m}^{2}(37 ; 53)$. A proportion of $23 \%$ had $\mathrm{E} / \mathrm{e}^{\prime}>15,67 \%$ had $\mathrm{E}^{\prime}<9,89 \%$ had LAVI $>34 \mathrm{~mL} / \mathrm{m}^{2}$ and $61 \%$ had LVH. Diastolic dysfunction was present in $52 \%$ while $35 \%$ did not fulfil the criteria. There were 5 patients with LVH and/or LAVI that did not have an $\mathrm{E} / \mathrm{e}^{\prime}>15$ or $\mathrm{E}^{\prime}<9$. Eleven patients (13\%) could not be classified due to missing echo variables.

Table 1 Copeptin as a predictor of all-cause mortality and/or heart failure hospitalisation in the 86 patients with HFPEF in KaRen

\begin{tabular}{|c|c|c|c|c|}
\hline \multirow[b]{2}{*}{ Parameter } & \multirow[b]{2}{*}{$\mathbf{n}$} & \multicolumn{3}{|c|}{$\begin{array}{l}\text { All-cause mortality or HF } \\
\text { hospitalisation }(n=36)\end{array}$} \\
\hline & & HR & $95 \% \mathrm{Cl}$ & p Value \\
\hline Copeptin & 86 & 1.56 & 1.03 to 2.38 & 0.037 \\
\hline $\begin{array}{l}\text { Copeptin (adjusted for } \\
\text { age) }\end{array}$ & 86 & 1.56 & 1.03 to 2.38 & 0.038 \\
\hline $\begin{array}{l}\text { Copeptin (adjusted for } \\
\text { gender) }\end{array}$ & 86 & 1.58 & 1.04 to 2.40 & 0.032 \\
\hline $\begin{array}{l}\text { Copeptin (adjusted for } \\
\text { GFR) }\end{array}$ & 85 & 1.69 & 0.94 to 3.01 & 0.078 \\
\hline $\begin{array}{l}\text { Copeptin (adjusted for } \\
N T \text {-proBNP) }\end{array}$ & 85 & 1.39 & 0.91 to 2.12 & 0.125 \\
\hline $\begin{array}{l}\text { Copeptin (adjusted for } \\
\text { age, gender and } \\
\text { NT-proBNP) }\end{array}$ & 85 & 1.40 & 0.92 to 2.14 & 0.119 \\
\hline $\begin{array}{l}\text { GFR, glomerular filtration } \\
\text { failturewith preserved ejec } \\
\text { Study; NT-proBNP, N-term }\end{array}$ & or & $\begin{array}{l}\text {, heart } \\
\text { ction; K } \\
\text { o-brain }\end{array}$ & $\begin{array}{l}\text { failture; HFPE } \\
\text { aRen, Karolins } \\
\text { natriuretic pep }\end{array}$ & $\begin{array}{l}\text { heart } \\
\text { a Rennes } \\
\text { le. }\end{array}$ \\
\hline
\end{tabular}

In the total HFPEF cohort copeptin was $13.56 \mathrm{pmol} / \mathrm{L}$ (8.56;20.55; table 2$)$. The levels did not differ between patients with or without diastolic dysfunction (figure 1). In addition copeptin was analysed in 62 healthy controls with a median age of 69 years and $44 \%$ females. Among controls $15 \%$ had hypertension, $13 \%$ type 2 diabetes and median weight was $74.5 \mathrm{~kg}$. Patients with HFPEF had significantly higher levels of copeptin compared to controls, $5.98 \mathrm{pmol} / \mathrm{L}(4.16 ; 9.42 ; \mathrm{p}$ value $<0.001)$. There was no difference in levels of copeptin between genders among patients with HFPEF, males 13.93 (8.43;20.43) versus females $13.06(8.70 ; 19.54 ; \mathrm{p}$ value $=0.483)$, whereas in controls there was, males $7.25(4.58 ; 12.06)$ versus females 4.69 (3.21;7.11;p value $=0.004)$.

Copeptin levels in patients with HFPEF did not correlate with assessed measurements of cardiac function, apart from IVRT $(-0.262 ; \mathrm{p}$ value $=0.021)$. Figure 2 displays relations between copeptin and echochardiographic measurements included in the definition of diastolic dysfunction.

In patients with diastolic dysfunction copeptin correlated only with $\mathrm{E} / \mathrm{e}^{\prime}(\mathrm{r}=0.459 ; \mathrm{p}$ value $=0.003)$ and $\mathrm{E}^{\prime}(\mathrm{r}=-0.402 ; \mathrm{p}$ value $=0.006)$ and when no diastolic dysfunction was present with LAVI $(r=0.390$; $\mathrm{p}$ value $=0.033$ ).

Both BMI and GFR correlated with copeptin in the overall HFPEF population $(\mathrm{r}=0.303 ; \mathrm{p}$ value $=0.005$ and $\mathrm{r}=-0.448 ; \mathrm{p}$ value $\leq 0.0001)$ as well as with BMI in patients with diastolic dysfunction $(\mathrm{r}=0.339$; $\mathrm{p}$ value $=0.023$ ). In patients without diastolic dysfunction there was a correlation with copeptin and GFR $(\mathrm{r}=-0.405 ; \mathrm{p}$ value $=0.026)$ but not with BMI. Copeptin correlated with NT-proBNP $(\mathrm{r}=0.223 ; \mathrm{p}$ value $=0.040)$ overall but not when the patients were divided according to diastolic function. There was no correlation between copeptin and age.

In the healthy control population copeptin correlated with age $(r=0.314 ; p$ value $=0.013)$ but not significantly with BMI (creatinine and NT-proBNP were not available).

Median follow-up time was 579 days (Q1;Q3 $276 ; 1178)$. No patient was lost to follow-up. The composite end point of HF hospitalisation or all-cause death occurred in 36 patients whereof 11 patients died during follow-up. In univariable analysis copeptin was a predictor of the composite end point (HR 1.56 (95\% CI 1.03 to $2.38 ; \mathrm{p}$ value $=0.037)$ ) and remained so after adjusting for age and gender (table 1) however, not for NT-proBNP or in the multivariable model. Increasing levels of copeptin did not predict the secondary end point mortality (HR 1.85 (95\% CI 0.87 to $3.94 ; \mathrm{p}$ value $=0.111)$ ). Diastolic dysfunction was not a predictor of the composite (HR $0.93(95 \%$ CI 0.46 to 1.88 ; p value $=0.836)$ or the secondary outcome (HR $0.77(95 \%$ CI 0.21 to 2.88; $p$ value $=0.701$ ).

\section{DISCUSSION}

Copeptin is elevated and predicts prognosis in HFREF but has been largely unexplored in HFPEF. Here we 
Table 2 Characteristics in the 86 patients in KaRen at 4-8 weeks visit

\begin{tabular}{|c|c|c|c|c|c|c|c|}
\hline \multirow[b]{3}{*}{ Parameter } & \multicolumn{7}{|c|}{ KaRen } \\
\hline & \multicolumn{2}{|c|}{$\begin{array}{l}\text { All patients* } \\
\mathrm{n}=86\end{array}$} & \multicolumn{2}{|c|}{$\begin{array}{l}\text { Diastolic } \\
\text { dysfunction } \\
n=45\end{array}$} & \multicolumn{2}{|c|}{$\begin{array}{l}\text { No diastolic } \\
\text { dysfunction } \\
n=30\end{array}$} & \multirow[b]{2}{*}{ p Value } \\
\hline & $\mathbf{n}$ & $(\%)$ & $\mathbf{n}$ & (\%) & $\mathbf{n}$ & $(\%)$ & \\
\hline \multicolumn{8}{|l|}{ Patient history } \\
\hline Age; median (Q1;Q3) & 73 & $(66 ; 79)$ & 73 & $(66 ; 80)$ & 72 & $(65 ; 72)$ & 0.360 \\
\hline Gender (male/female) & $42 / 44$ & $(49 / 51)$ & $19 / 26$ & $(42 / 58)$ & $16 / 14$ & $(53 / 47)$ & 0.358 \\
\hline Hypertension & 68 & (79) & 37 & (82) & 25 & (83) & 1.000 \\
\hline COPD & 14 & (16) & 8 & (18) & 5 & (17) & 1.000 \\
\hline T2DM & 27 & (31) & 17 & (38) & 8 & (27) & 0.454 \\
\hline Coronary heart disease & 29 & (34) & 19 & (42) & 8 & (27) & 0.222 \\
\hline Atrial fibrillation & 49 & (57) & 24 & (53) & 19 & (63) & 0.477 \\
\hline NYHA class I & 19 & (22) & 10 & (22) & 5 & (17) & 0.758 \\
\hline NYHA class II & 47 & (55) & 27 & (60) & 18 & (60) & \\
\hline NYHA class III & 20 & (23) & 8 & (18) & 7 & (23) & \\
\hline \multicolumn{8}{|l|}{ Measurements } \\
\hline BMl $\left(\mathrm{kg} / \mathrm{m}^{2}\right)$ & 29 & $(25 ; 33)$ & 29 & $(27 ; 33)$ & 27 & $(24 ; 32)$ & 0.124 \\
\hline Systolic blood pressure (mm Hg) & 140 & $(90 ; 210)$ & 145 & $(130 ; 150)$ & 148 & $(125 ; 155)$ & 0.944 \\
\hline Diastolic blood pressure $(\mathrm{mm} \mathrm{Hg})$ & 80 & $(70 ; 85)$ & 80 & $(70 ; 85)$ & 83 & $(75 ; 85)$ & 0.152 \\
\hline Heart rate $(\mathrm{bpm})$ & 70 & $(60 ; 80)$ & 69 & $(60 ; 78)$ & 72 & $(63 ; 84)$ & 0.210 \\
\hline \multicolumn{8}{|l|}{ Treatment } \\
\hline ARB & 28 & (33) & 17 & (38) & 7 & (23) & 0.216 \\
\hline ACE-inhibitor & 42 & (49) & 21 & (47) & 16 & (53) & 0.641 \\
\hline Thiazid diuretics & 14 & (16) & 7 & (16) & 5 & (17) & 1.000 \\
\hline Potassium sparing diuretics & 18 & (21) & 12 & (27) & 4 & (13) & 0.250 \\
\hline Loop diuretics & 63 & (73) & 34 & (76) & 23 & (77) & 1.000 \\
\hline$\beta$-blocker & 69 & (80) & 38 & (84) & 21 & (70) & 0.159 \\
\hline Anticoagulants & 47 & (55) & 21 & (47) & 20 & (67) & 0.103 \\
\hline Pacemaker & 20 & (23) & 9 & (20) & 6 & (20) & 1.000 \\
\hline \multicolumn{8}{|l|}{ ECHO parameters } \\
\hline LVEF (\%) & 64 & $(58 ; 68)$ & 63 & $(60 ; 68)$ & 64 & $(58 ; 66)$ & 0.847 \\
\hline LAVI $\left(\mathrm{mL} / \mathrm{m}^{2}\right)$ & 43.3 & $(37.2 ; 52.8)$ & 41.7 & $(38.2 ; 50.8)$ & 44.7 & $(37.0 ; 55.0)$ & 0.565 \\
\hline Left atrial volume $(\mathrm{mL})$ & 86.5 & $(75 ; 104)$ & 84.0 & 84.0 & 88.5 & $(74 ; 106)$ & 0.726 \\
\hline Left ventricular mass index $\left(\mathrm{g} / \mathrm{m}^{2}\right)$ & 115 & $(95 ; 142)$ & 115 & $(95 ; 142)$ & 123 & $(92 ; 144)$ & 1.000 \\
\hline Male & 125 & $(102 ; 157)$ & 143 & $(102 ; 157)$ & 121 & $(81 ; 146)$ & 0.362 \\
\hline Female & 109 & $(94 ; 136)$ & 102 & $(95 ; 133)$ & 138 & $(92 ; 144)$ & 0.379 \\
\hline LVEDd (mm) & 47 & $(43 ; 53)$ & 47 & $(43 ; 53)$ & 47 & $(42 ; 54)$ & 0.948 \\
\hline E/A ratio & 1.3 & $(0.9 ; 2.5)$ & 1.2 & $(0.9 ; 2.0)$ & 1.4 & $(1.1 ; 3.4)$ & 0.329 \\
\hline E/e' ratio & 10.8 & $(8.3 ; 14.0)$ & 13.6 & $(10.0 ; 18.2)$ & 7.9 & $(7.1 ; 9.6)$ & $<0.001$ \\
\hline$E^{\prime}(\mathrm{cm} / \mathrm{s})$ & 8.0 & $(7.0 ; 10.0)$ & 7.5 & $(6.0 ; 8.0)$ & 10.5 & $(9.5: 12)$ & $<0.001$ \\
\hline IVRT (diastole) & 94 & $(77 ; 113)$ & 102 & $(79 ; 119)$ & 86 & $(72 ; 102)$ & 0.036 \\
\hline Mitral VTI & 23 & $(16 ; 30)$ & 26 & $(22 ; 31)$ & 17 & $(13 ; 24)$ & $<0.001$ \\
\hline E-wave deceleration time (ms) & 203 & $(156 ; 228)$ & 205 & $(177 ; 225)$ & 164 & $(139 ; 227)$ & 0.054 \\
\hline \multicolumn{8}{|l|}{ Biochemistry } \\
\hline Copeptin (pmol/L) & 13.56 & $(8.56 ; 20.55)$ & 11.5 & $(7.6 ; 20.4)$ & 14.7 & $(9.2 ; 20.3)$ & 0.313 \\
\hline Males & 13.93 & $(8.43 ; 20.43)$ & 13.7 & $(7.6 ; 28.3)$ & 13.6 & $(8.8 ; 20.0)$ & 0.831 \\
\hline Females & 13.06 & $(8.70 ; 19.54)$ & 10.9 & $(6.8 ; 16.7)$ & 16.0 & $(11.9 ; 24.4)$ & 0.127 \\
\hline NT-proBNP (ng/L) & 1000 & $(469 ; 2330)$ & 574 & $(385 ; 2330)$ & 1320 & $(824 ; 1830)$ & 0.194 \\
\hline Glomerular filtration rate $\left(\mathrm{mL} / \mathrm{min} / 1.73 \mathrm{~m}^{2}\right)$ & 68 & $(51 ; 81)$ & 68 & $(50 ; 80)$ & 77 & $(56 ; 82)$ & 0.375 \\
\hline
\end{tabular}

Continuous variables are presented as median and lower and upper quartiles $(\mathrm{Q} 1 ; \mathrm{Q} 3)$ and categorical variables as numbers $(\mathrm{n})$ and percentages when not otherwise stated.

${ }^{*}$ Eleven patients not categorised regarding diastolic dysfunction due to missing echo variables.

ARB, angiotensin receptor blocker; BMI, body mass index; COPD, chronic obstructive pulmonary disease; IVRT, isovolumetric relaxation time; LAVI, left atrial volume index; LVEF, left ventricular ejection fraction; LVEDd, left ventricular end diastolic diameter; Mitral VTI, mitral to aortic velocity-time integral ratio; NT-proBNP, N-terminal pro-brain natriuretic peptide; NYHA, New York Heart Association; T2DM, type 2 diabetes mellitus.

show that in patients with HFPEF, copeptin is elevated, correlates with NT-proBNP but not with markers of diastolic dysfunction, and is a prognostic predictor of the composite end point (HF hospitalisation or mortality) however blunted by NT-proBNP. This suggest that the HFPEF syndrome is associated with activation of 


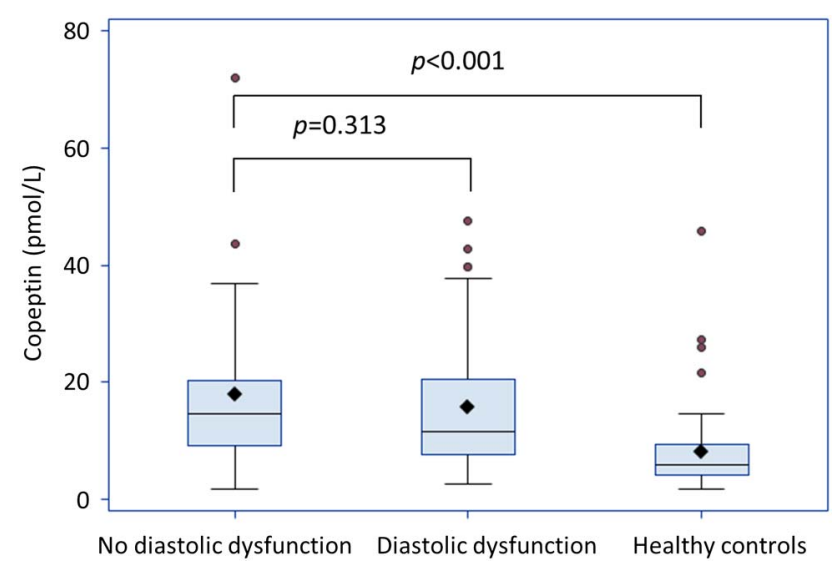

Figure 1 Copeptin levels in KaRen patients divided according to diastolic dysfunction and in healthy controls presented as boxplots displaying IQR, median (-), mean ( $\downarrow)$ and outliers (๑). Whiskers represent maximum observation within 1.5 IQR above the 75th centile.

copeptin however not related to measurements of an impaired diastolic function indicating that the HFPEF syndrome may be reflected more by neurohormonal activation than by diastolic dysfunction.

In the present material copeptin levels were significantly higher among patients with HFPEF (13.56 pmol/ L (8.56; 20.55)) compared to a control population with similar age and gender distribution $(5.98 \mathrm{pmol} / \mathrm{L}$ (4.16; 9.42))

The elevated copeptin levels in the KaRen HFPEF cohort contrast to findings in the CIBIS-ELD trial in which copeptin levels among patients with HFPEF were in the normal range $(3.7 \mathrm{pmol} / \mathrm{L}(2.0,8.6))$. In fact the KaRen patients had copeptin levels similar to patients with HFREF participating in the randomised controlled CIBIS-ELD trial $(10.8 \mathrm{pmol} / \mathrm{L}(5.6 ; 18.2)) .^{22}$ This may be explained by the different inclusion criteria in the two studies with a recent acute exacerbation of $\mathrm{HF}$ required for inclusion criteria in KaRen and instead constituting an exclusion criterion in CIBIS-ELD ${ }^{14}$ that mainly recruited chronic patients with HF. Moreover, a large proportion of KaRen patients were sicker and with more comorbidities than in the CIBIS-ELD trial. Furthermore the prevalence of diabetes, a condition associated with elevated levels of copeptin, was present in $31 \%$ of patients in KaRen and in $19 \%$ and $29 \%$ of CIBIS-ELD patients with HFPEF and HFREF, respectively. ${ }^{23}$ This may indicate that the elevated copeptin levels in KaRen also, to some extent, may relate to common comorbid conditions in HFPEF such as diabetes, which indeed are also implicated in HFPEF pathophysiology. ${ }^{24}$

Also Mason et a $t^{25}$ have demonstrated high levels of copeptin in HFPEF (mean $22 \mathrm{pmol} / \mathrm{L}$ (range 5-154)) comparable to patients with undifferentiated HF (mean $21 \mathrm{pmol} / \mathrm{L}$ (range 5-154))indicating increased hormonal activation. In the same report individuals without $\mathrm{HF}$ had a mean copeptin level of $16 \mathrm{pmol} / \mathrm{L}$ (5-184). The high copeptin levels (although reported as mean and not median which make a direct comparison difficult) may partly be explained by the study design as this was a screening study and no exclusions were made on the basis of cognitive capacity, comorbidities or immobility. Further participants defined as having no HF actually had signs of decompensation such as peripheral oedema in $40 \%$ and lung crackles in $17 \%$ of the cases. ${ }^{26}$

Activation of the vasopressin axis was one the first neurohoromonal axis described to be involved in the pathogenesis of $\mathrm{HF}^{27}$ In addition to the well-known haemodynamic effects of vasopressin, vasoconstriction and water retention, the hormone has been proposed to exert direct effects on the myocardium. These effects may be detrimental in a long-term perspective leading to left ventricular hypertrophy and remodelling in turn resulting in negative effects on myocardial contractility. ${ }^{28} 29$ Noteworthy in this context are findings in patients with myocardial infarction and slightly depressed LVEF where copeptin has been associated with left ventricular dysfunction and remodelling. ${ }^{30}$ This is in contrast to the present results in the KaRen population with HFPEF as we did not find correlations between copeptin and measurements of cardiac function. The discrepancy may relate to differences in study design, including the measurements of cardiac function used and sample size, but more importantly differences in study population as we studied copeptin a distinct HFPEF population.

Interestingly levels of copeptin did not differ in patients with HFPEF with and without diastolic dysfunction. This may relate to the small sample size but similar baseline characteristics and comorbidities between the two groups further strengthen the speculation that elevated copeptin levels in this cohort is a marker of hormonal activation accompanied by other conditions such as renal impairment, chronic lung diseases, anaemia, cancer, liver disease common in the HFPEF population ${ }^{5}$ rather than a marker of the by echocardiography defined condition diastolic dysfunction. Indeed copeptin did correlate with renal function, BMI as well as NT-proBNP but not with measurements of structural remodelling (LV hypertrophy or dilated left atrium) or raised cardiac filling pressures on echocardiography.

Of note in this context is that the patients in KaRen categorised as not having diastolic dysfunction had similar or even higher levels of copeptin and NT-proBNP however the difference not was statistically significant. This may indicate that diastolic dysfunction according to the present definition not necessarily reflect increased neurohormonal activation.

In the present HFPEF population copeptin, in unadjusted analyses, was a predictor of the composite of HF hospitalisations and mortality but not of mortality alone. Previously copeptin has been presented as a prognostic marker in HF in general ${ }^{10-12} 30$ however not, at least to the best of our knowledge, explicitly in HFPEF. The present results indicate a prognostic value of 

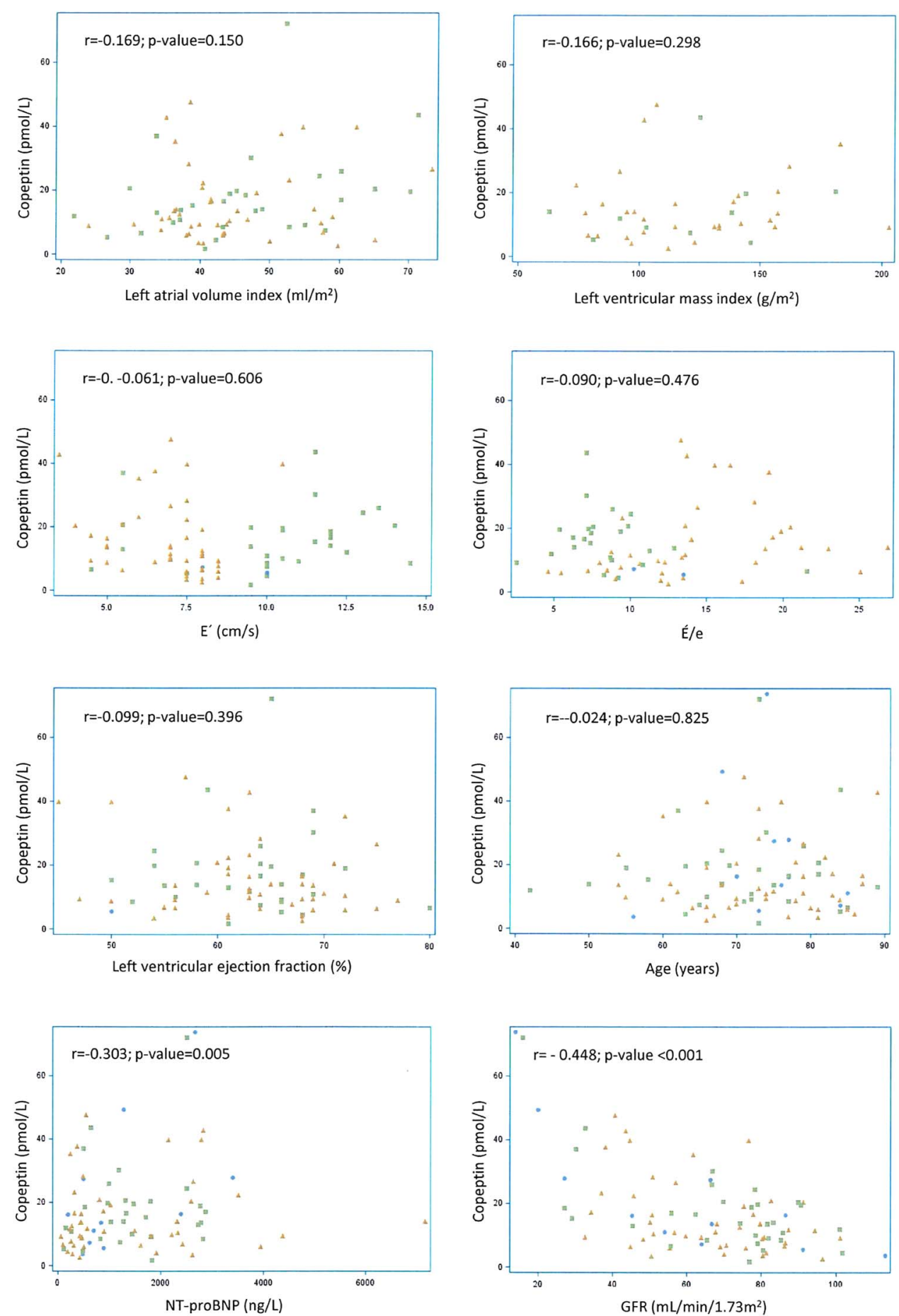

Figure 2 Correlation between copeptin and biochemical markers, echocardiographic variables and clinical variables in the 86 patients in KaRen biochemistry substudy.

copeptin in HFPEF however blunted when adjusted for the well-established HF biomarker NT-proBNP. Thus copeptin alone may not be a biomarker to predict prognosis in HFPEF but it may add information on underlying mechanisms in this syndrome. This further supports the speculation that hormonal activation, in combination with comorbidities related to HFPEF, are of importance for prognosis and disease progression however not directly related to diastolic dysfunction, at least by the present definition. 
Despite a proportionally high event rate the potential lack of power due to the small sample size is a limitation and it is therefore important to further study the potential role of copeptin as a prognostic marker in larger patient materials with HFPEF. In addition to the lack of power the sample size may have precluded the possibility to discover any differences in subgroup analyses. KaRen was designed prior to the $2012 \mathrm{ESC}$ guidelines requiring structural heart disease or diastolic dysfunction on echocardiography. For inclusion, we required acute decompensated HF together with specific Framingham signs and symptoms of $\mathrm{HF}$ and had a range of exclusion criteria. ${ }^{14}$ Nevertheless, with an NT-proBNP cut-off of $300 \mathrm{ng} / \mathrm{L}$, we cannot exclude that some patients may not have had HF. However, median NT-proBNP levels were high and indeed higher in patients without diastolic dysfunction on echocardiography. Selection bias and measurements errors may have confounded the results.

A comparison with echocardiographic measurement's relation to copeptin in the control group would have been an advantage but was not available. Further serial measurements may have provided additional information on for example markers of ventricular remodelling such as temporal changes in end diastolic or systolic volumes which were associated with copeptin in the study by Kelly et $a l^{30}$ Categorising patients with atrial fibrillation as having diastolic dysfunction according to echocardiography may be difficult. As there is no established cut-off specifically for patients with atrial fibrillation they were categorised according to the same criteria as those in sinus rhythm.

\section{CONCLUSION}

The pathophysiology behind HFPEF, a condition associated with a poor prognosis, is still to a large extent unknown and studies on vasoactive hormones may shed some light. In the present cohort of patients with HFPEF copeptin levels were increased compared to controls and predicted the composite end point of future HF hospitalisations and mortality, however blunted by NT-proBNP. Copeptin was not correlated to measures of cardiac function including diastolic function. Our findings suggest that the HFPEF syndrome is associated with activation of vasopressin and that the pathophysiology in HFPEF is reflected by neurohormonal activation rather than by diastolic dysfunction. This is important as new information on the underlying mechanisms in HFPEF is crucial for the much needed development of novel treatment options for these patients.

Acknowledgements The authors are grateful to Gunilla Förstedt at Karolinska University hospital for blood sampling and patient care, Kambiz Shahgaldi and Maria Westerlind for echocardiogram assessments and to Eva Wallgren for laboratory analysis.

Contributors $\mathrm{CH}$ perfomed statistics, $\mathrm{CH}$, LM and LHL wrote the manuscript. All authors have actively contributed to the conductance of the study and reviewed the manuscript.
Funding This work was supported by grants from Center for Gender Medicine Karolinska Institutet, Stockholm Sweden (to $\mathrm{CH}$ ) and The Prospective KaRen study was supported in part by grants from Fédération Française de Cardiologie/Société Française de Cardiologie, France and Medtronic Bakken Research Center, Maastricht, the Netherlands. LHL was supported by the Swedish Research Council (grant 2013-23897-104604-23) Swedish Heart Lung Foundation (grants 20080409 and 20100419) and the Stockholm County Council (grants 00556-2009 and 20110120). CL was supported by the Swedish Heart Lung Foundation (grants 20080498 and 20110406) and the Stockholm County Council (grants 20090376 and 20110610). No funding agency had any role in the design and conduct of the study, in the collection, management, analysis or interpretation of the data, or in the preparation, review, or approval of the manuscript.

Competing interests $\mathrm{CH}$ received consulting fees from Novartis and speaker and honoraria from MSD. LHL received research grants and speaker and honoraria from AstraZeneca, consulting honoraria from Novartis and St Jude Medical, and research grants from Boston Scientific. CL was the principal investigator of REVERSE, a CRT study sponsored by Medtronic research grants, speaker honoraria and consulting fees from Medtronic, speaker honoraria and consulting fees from St. Jude Medical. ED received speaker honoraria and consulting fees from Novartis, AstraZeneca. JCD received research grants, speaker honoraria and consulting fees from Medtronic and St Jude Medical. LM received Swedish Heart-Lung Foundation, the Swedish Diabetes Foundation, MSD, Bayer AG, Sanofi-Aventis, and personal fees from MSD, Sanofi-Aventis, Novartis, Bayer-Schering, AstraZeneca, Lilly and Roche, Novo Nordisk.

\section{Patient consent Obtained.}

Provenance and peer review Not commissioned; externally peer reviewed.

Open Access This is an Open Access article distributed in accordance with the Creative Commons Attribution Non Commercial (CC BY-NC 4.0) license, which permits others to distribute, remix, adapt, build upon this work noncommercially, and license their derivative works on different terms, provided the original work is properly cited and the use is non-commercial. See: http:// creativecommons.org/licenses/by-nc/4.0/

\section{REFERENCES}

1. Owan TE, Hodge DO, Herges RM, et al. Trends in prevalence and outcome of heart failure with preserved ejection fraction. $N$ Engl $J$ Med 2006;355:251-9.

2. Bhatia RS, Tu JV, Lee DS, et al. Outcome of heart failure with preserved ejection fraction in a population-based study. $N$ Engl $J$ Med 2006;355:260-9.

3. Somaratne JB, Berry C, McMurray JJ, et al. The prognostic significance of heart failure with preserved left ventricular ejection fraction: a literature-based meta-analysis. Eur $J$ Heart Fail 2009;11:855-62.

4. Hogg K, Swedberg K, McMurray J. Heart failure with preserved left ventricular systolic function; epidemiology, clinical characteristics, and prognosis. J Am Coll Cardiol 2004;43:317-27.

5. Lam CS, Donal E, Kraigher-Krainer E, et al. Epidemiology and clinical course of heart failure with preserved ejection fraction. Eur $J$ Heart Fail 2011;13:18-28.

6. Meta-analysis Global Group in Chronic Heart Failure (MAGGIC). The survival of patients with heart failure with preserved or reduced left ventricular ejection fraction: an individual patient data meta-analysis. Eur Heart J 2012;33:1750-7.

7. Campbell RT, Jhund PS, Castagno D, et al. What have we learned about patients with heart failure and preserved ejection fraction from DIG-PEF, CHARM-preserved, and I-PRESERVE? J Am Coll Cardiol 2012;60:2349-56.

8. Francis GS, Benedict C, Johnstone DE, et al. Comparison of neuroendocrine activation in patients with left ventricular dysfunction with and without congestive heart failure. A substudy of the Studies of Left Ventricular Dysfunction (SOLVD). Circulation 1990;82:1724-9.

9. Morgenthaler NG, Struck J, Alonso C, et al. Assay for the measurement of copeptin, a stable peptide derived from the precursor of vasopressin. Clin Chem 2006;52:112-19.

10. Stoiser B, Mörtl D, Hülsmann M, et al. Copeptin, a fragment of the vasopressin precursor, as a novel predictor of outcome in heart failure. Eur J Clin Invest 2006;36:771-8. 
11. Maisel A, Xue Y, Shah $\mathrm{K}$, et al. Increased 90-day mortality in patients with acute heart failure with elevated copeptin: secondary results from the Biomarkers in Acute Heart Failure (BACH) study. Circ Heart Fail 2011;4:613-20.

12. Gegenhuber A, Struck J, Dieplinger B, et al. Comparative evaluation of B-type natriuretic peptide, mid-regional pro-A-type natriuretic peptide, mid-regional pro-adrenomedullin, and Copeptin to predict 1-year mortality in patients with acute destabilized heart failure. $J$ Card Fail 2007;13:42-9.

13. Butler J, Fonarow GC, Zile MR, et al. Developing therapies for heart failure with preserved ejection fraction: current state and future directions. JACC Heart Fail 2014;2:97-112.

14. Donal E, Lund LH, Linde C, et al. Rationale and design of the Karolinska-Rennes (KaRen) prospective study of dyssynchrony in heart failure with preserved ejection fraction. Eur J Heart Fail 2009;11:198-204.

15. Donal E, Lund LH, Oger E, et al. Baseline characteristics of patients with heart failure and preserved ejection fraction included in the Karolinska Rennes (KaRen) study. Arch Cardiovasc Dis 2014;107:112-21.

16. Bartnik M, Malmberg K, Hamsten A, et al. Abnormal glucose tolerance-a common risk factor in patients with acute myocardial infarction in comparison with population-based controls. J Intern Med 2004;256:288-97.

17. Iversen K, Gøtze JP, Dalsgaard M, et al. Risk stratification in emergency patients by copeptin. BMC Med 2014;12:80.

18. Terzic D, Johansson-Fallgren AS, Ragnarsson O, et al. Evaluation of a sensitive copeptin assay for clinical measurement. Open Clin Chem J 2012;5:21-6.

19. Prontera C, Emdin M, Zucchelli GC, et al. Analytical performance and diagnostic accuracy of a fully-automated electrochemiluminescent assay for the $\mathrm{N}$-terminal fragment of the pro-peptide of brain natriuretic peptide in patients with cardiomyopathy: comparison with immunoradiometric assay methods for brain natriuretic peptide and atrial natriuretic peptide. Clin Chem Lab Med 2004;42:37-44.

20. Levey AS, Stevens LA, Schmid CH, et al. A new equation to estimate glomerular filtration rate. Ann Intern Med 2009;150:604-12.
21. McMurray JJ, Adamopoulos S, Anker SD, et al. ESC Guidelines for the diagnosis and treatment of acute and chronic heart failure 2012: The Task Force for the Diagnosis and Treatment of Acute and Chronic Heart Failure 2012 of the European Society of Cardiology. Developed in collaboration with the Heart Failure Association (HFA) of the ESC. Eur Heart $J$ 2012;33: 1787-847.

22. Loncar G, von Haehling S, Tahirovic E, et al. Effect of beta blockade on natriuretic peptides and copeptin in elderly patients with heart failure and preserved or reduced ejection fraction: results from the CIBIS-ELD trial. Clin Biochem 2012;45:117-22.

23. Enhörning S, Wang TJ, Nilsson PM, et al. Plasma copeptin and the risk of diabetes mellitus. Circulation 2010;121:2102-8.

24. Paulus WJ, Tschöpe C. A novel paradigm for heart failure with preserved ejection fraction: comorbidities drive myocardial dysfunction and remodeling through coronary microvascular endothelial inflammation. J Am Coll Cardiol 2013;62: 263-71.

25. Mason JM, Hancock HC, Close $\mathrm{H}$, et al. Utility of biomarkers in the differential diagnosis of heart failure in older people: findings from the heart failure in care homes ( $\mathrm{HFinCH}$ ) diagnostic accuracy study. PLOS ONE 2013;8:e53560.

26. Hancock $\mathrm{HC}$, Close $\mathrm{H}$, Mason JM, et al. High prevalence of undetected heart failure in long-term care residents: findings from the Heart Failure in Care Homes (HFinCH) study. Eur J Heart Fail 2013;15:158-65.

27. Francis GS, Goldsmith SR, Levine TB, et al. The neurohumoral axis in congestive heart failure. Ann Intern Med 1984;101: 370-7.

28. Goldsmith SR, Gheorghiade M. Vasopressin antagonism in heart failure. J Am Coll Cardiol 2005;46:1785-91.

29. Yamazaki T, Izumi Y, Nakamura Y, et al. Tolvaptan improves left ventricular dysfunction after myocardial infarction in rats. Circ Heart Fail 2012:5:794-802.

30. Kelly D, Squire IB, Khan SQ, et al. C-terminal provasopressin (copeptin) is associated with left ventricular dysfunction, remodeling, and clinical heart failure in survivors of myocardial infarction. $J$ Card Fail 2008;14:739-45. 